\title{
Sensitive Top-Down Proteomics Analysis of Low Number of Mammalian Cells Using a Nanodroplet Sample Processing Platform
}

Mowei Zhou, ${ }^{1}+$ Naomi Uwugiaren, ${ }^{2}+$ Sarah M. Williams, ${ }^{1}$ Ronald J. Moore, ${ }^{3}$ Rui Zhao, ${ }^{1}$ David Goodlett, ${ }^{2,4}$ Irena Dapic, ${ }^{2}$ Ljiljana Paša-Tolić, ${ }^{1}$ Ying Zhu ${ }^{1 *}$

1. Environmental Molecular Sciences Laboratory, Pacific Northwest National Laboratory, Richland, WA 99354, United States

2. International Centre for Cancer Vaccine Science, University of Gdansk, Gdansk, Poland

3. Biological Sciences Division, Pacific Northwest National Laboratory, Richland, Washington 99354, United States

4. Department of Microbial Pathogenesis, School of Dentistry, University of Maryland, Baltimore, Maryland 21201, United States.

\$ the authors contributed equally

* corresponding: ying.zhu@pnnl.gov

\section{List of contents}

Figure S1. Venn diagrams showing the overlap of identified proteoforms and proteins among triplicate nanoPOTS top-down analysis of $\sim 100 \mathrm{HeLa}$ cells using (a) DDM, (b) Invitrosol, and (c) ProteaseMAX in the extraction buffers.

Figure S2. Venn diagrams showing the overlap of identified proteoforms and proteins among triplicate nanoPOTS top-down analysis of $\sim 100 \mathrm{HeLa}$ cells using (a) $8 \mathrm{M}$ urea+ $0.1 \%$ DDM, (b) $0.01 \%$ PEG $20000+0.1 \%$ DDM, (c) $0.5 \%$ DDM, and (d) $0.1 \%$ DDM in the extraction buffers.

Figure S3. Venn diagrams showing the overlap of detected proteoforms and proteins among triplicate nanoPOTS top-down analysis of (a) $74 \pm 7$ cells, (b) $217 \pm 79$ cells, and (c) $769 \pm 22$ cells.

Figure S4. Mass and intensity distribution for proteoforms identified by triplicate nanoPOTS top-down analysis of $769 \pm 22$ cells.

Figure S5. Representative LCMS feature map of nanoPOTS top-down analysis of $777 \mathrm{HeLa}$ cells.

Figure S6. Representative spectra showing detection of 30-60 kDa proteins using TOF mass spectrometer.

Figure S7. Tandem mass spectra for the proteoform identified as N-myristoylated brain acid soluble protein (UniProt ID: P80723).

Figure S8. Tandem mass spectra for the proteoform identified as60S acidic ribosomal protein P2 (UniProt ID: P05387). 
Figure S9. Tandem mass spectra for the proteoform identified as N-acetylated and iron-bound thymosin beta-4 (UniProt ID: P62328).

(a) DDM
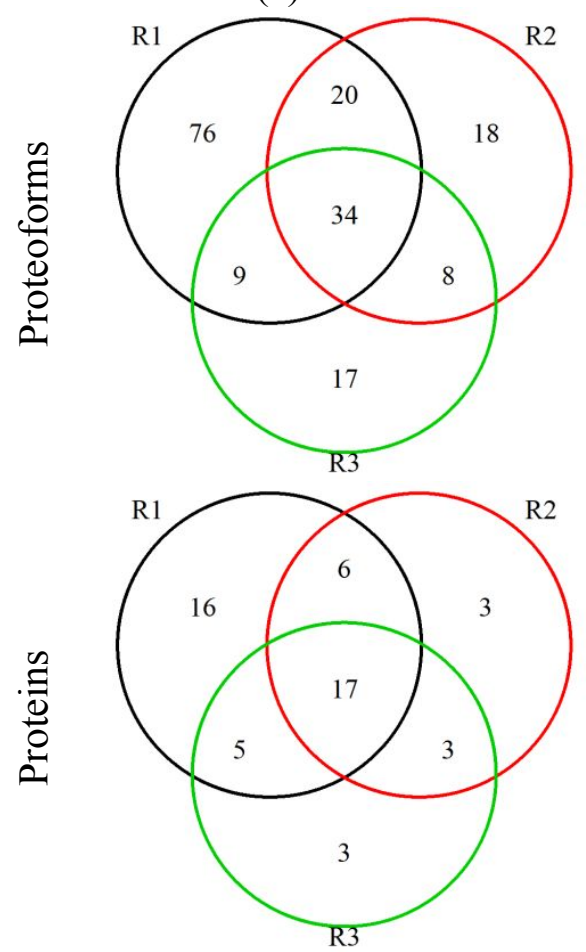

(b) Invitrosol
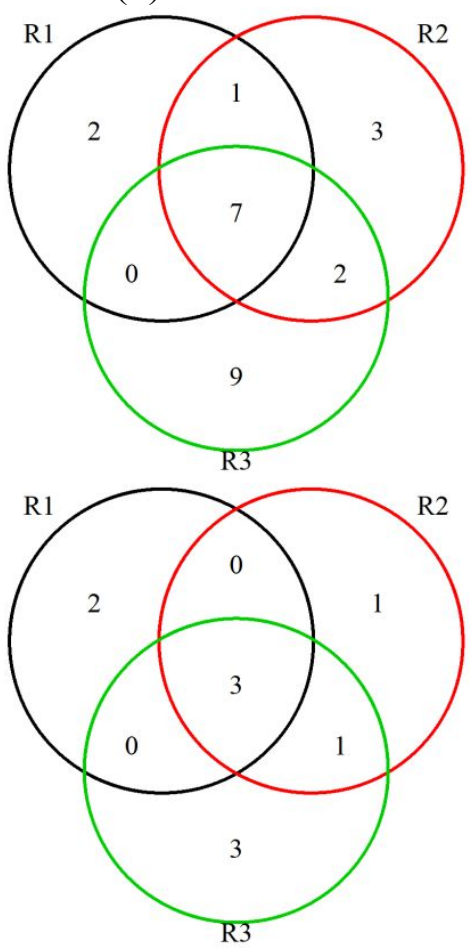

(c) ProteaseMAX
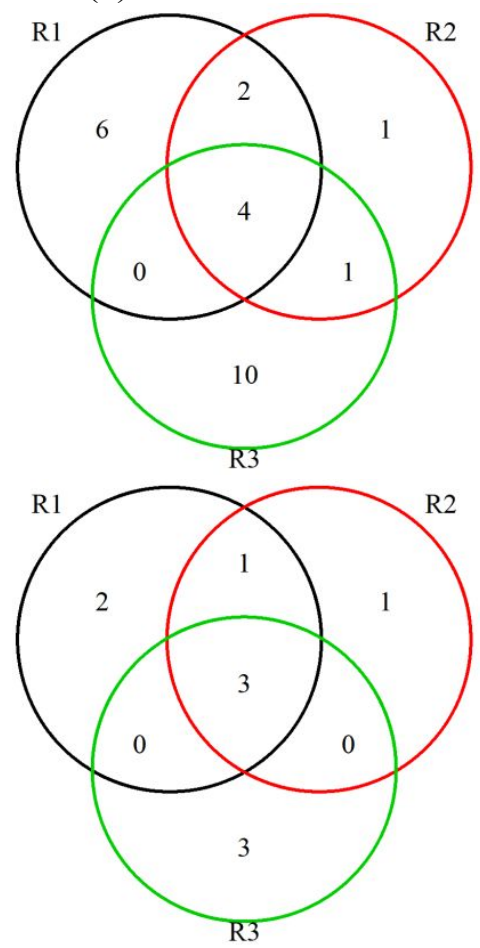

Figure S1. Venn diagrams showing the overlap of identified proteoforms and proteins among triplicate nanoPOTS top-down analysis of $\sim 100 \mathrm{HeLa}$ cells using (a) DDM, (b) Invitrosol, and (c) ProteaseMAX in the extraction buffers. The control samples were not shown because only a single protein was detected in 1 out of 3 replicates. 


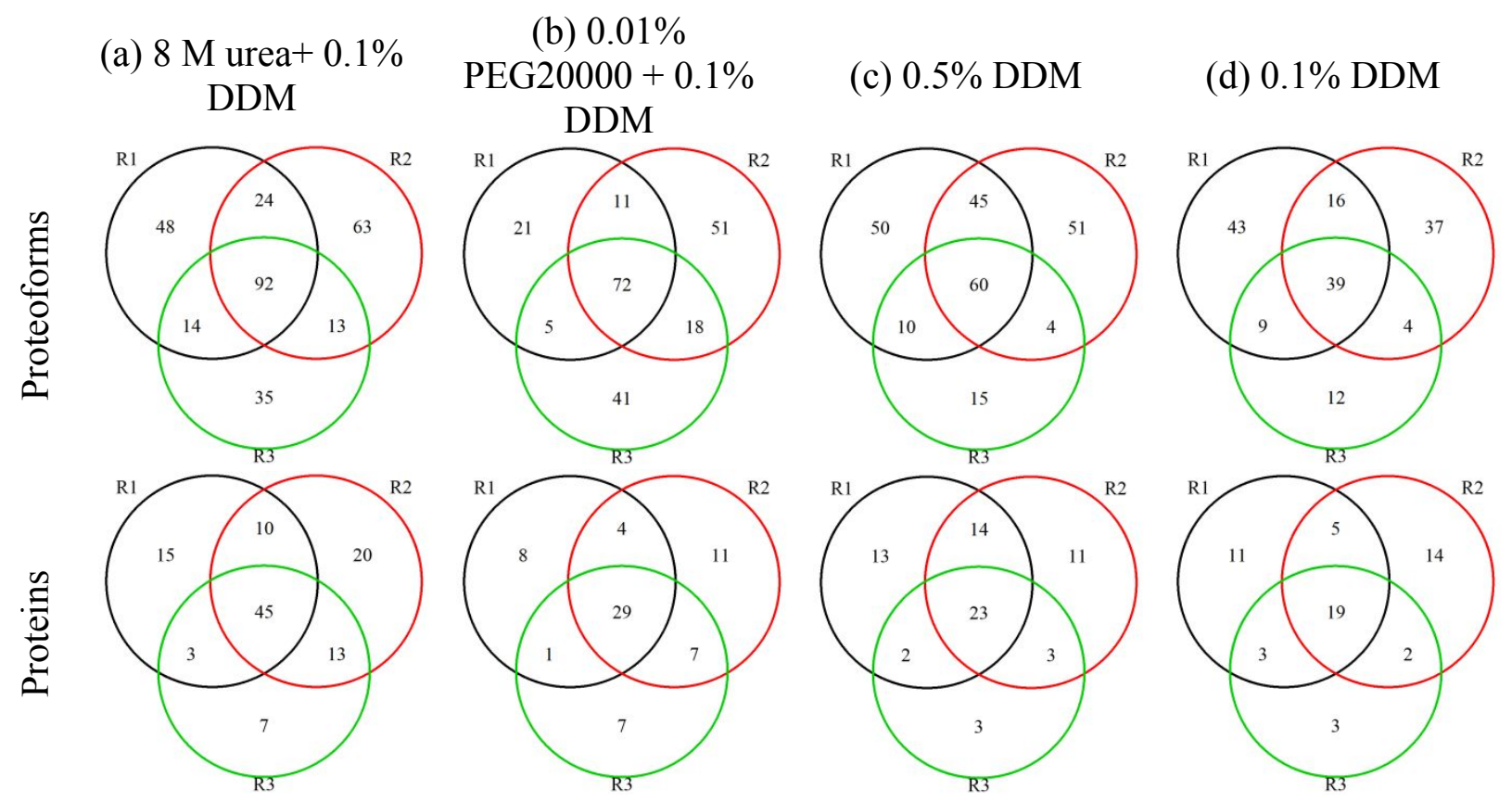

Figure S2. Venn diagrams showing the overlap of identified proteoforms and proteins among triplicate nanoPOTS top-down analysis of $\sim 100 \mathrm{HeLa}$ cells using (a) $8 \mathrm{M}$ urea $+0.1 \%$ DDM, (b) $0.01 \%$ PEG $20000+0.1 \%$ DDM, (c) $0.5 \%$ DDM, and (d) $0.1 \%$ DDM in the extraction buffers. 


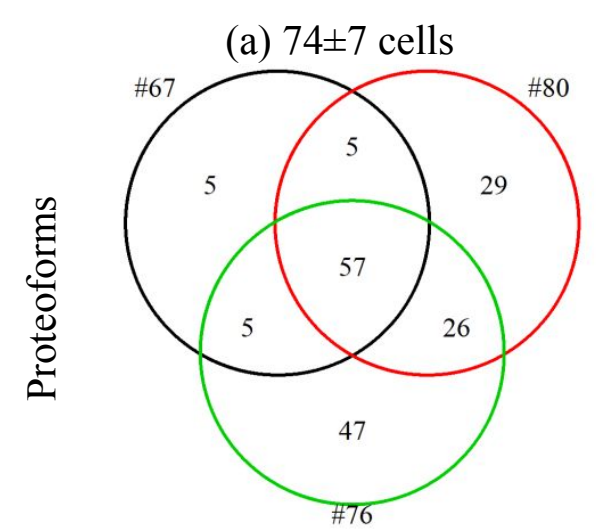

(b) $217 \pm 79$ cells
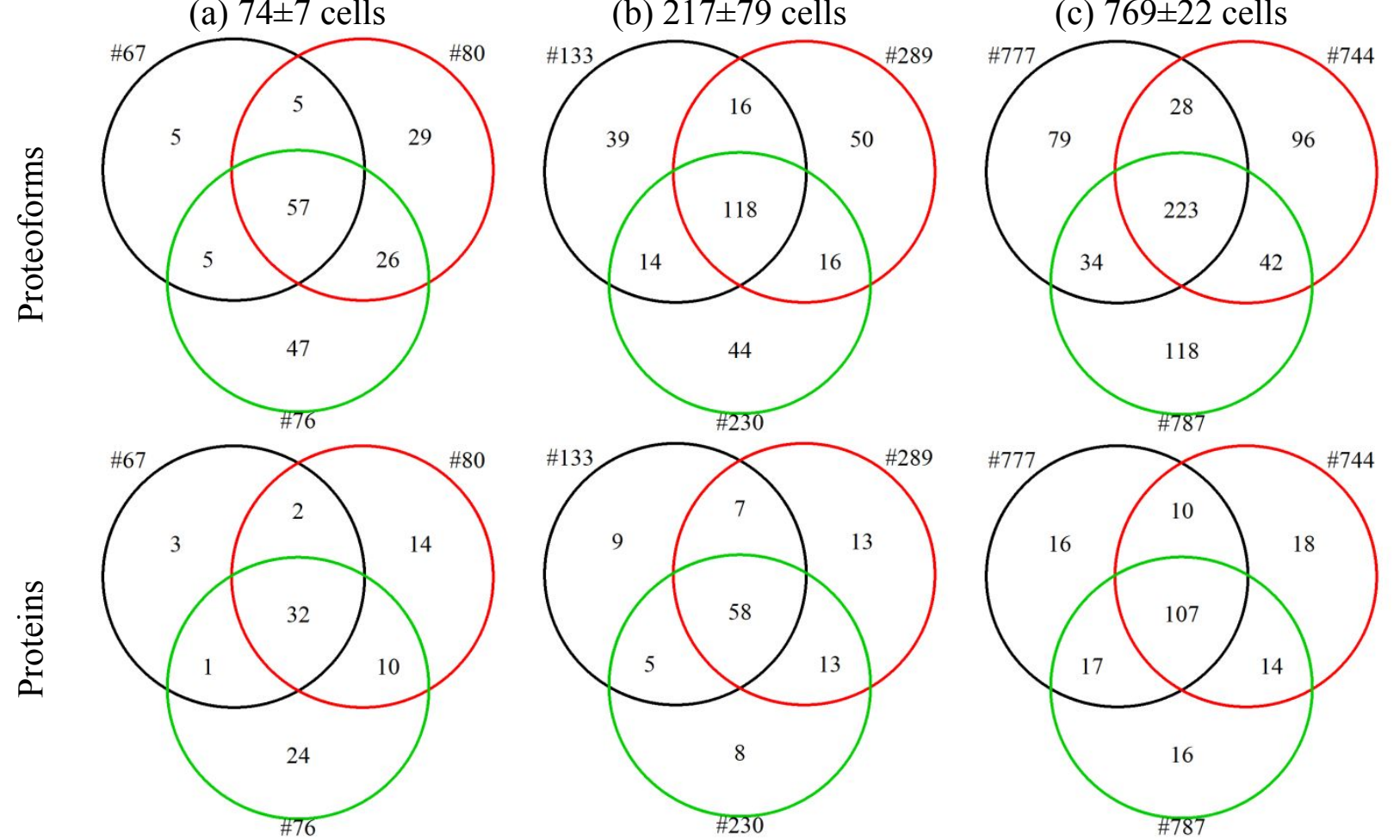

Figure S3. Venn diagrams showing the overlap of detected proteoforms and proteins among triplicate nanoPOTS top-down analysis of (a) $74 \pm 7$ cells, (b) $217 \pm 79$ cells, and (c) $769 \pm 22$ cells. Cell counts for individual experiments are labeled next to the corresponding circles. 


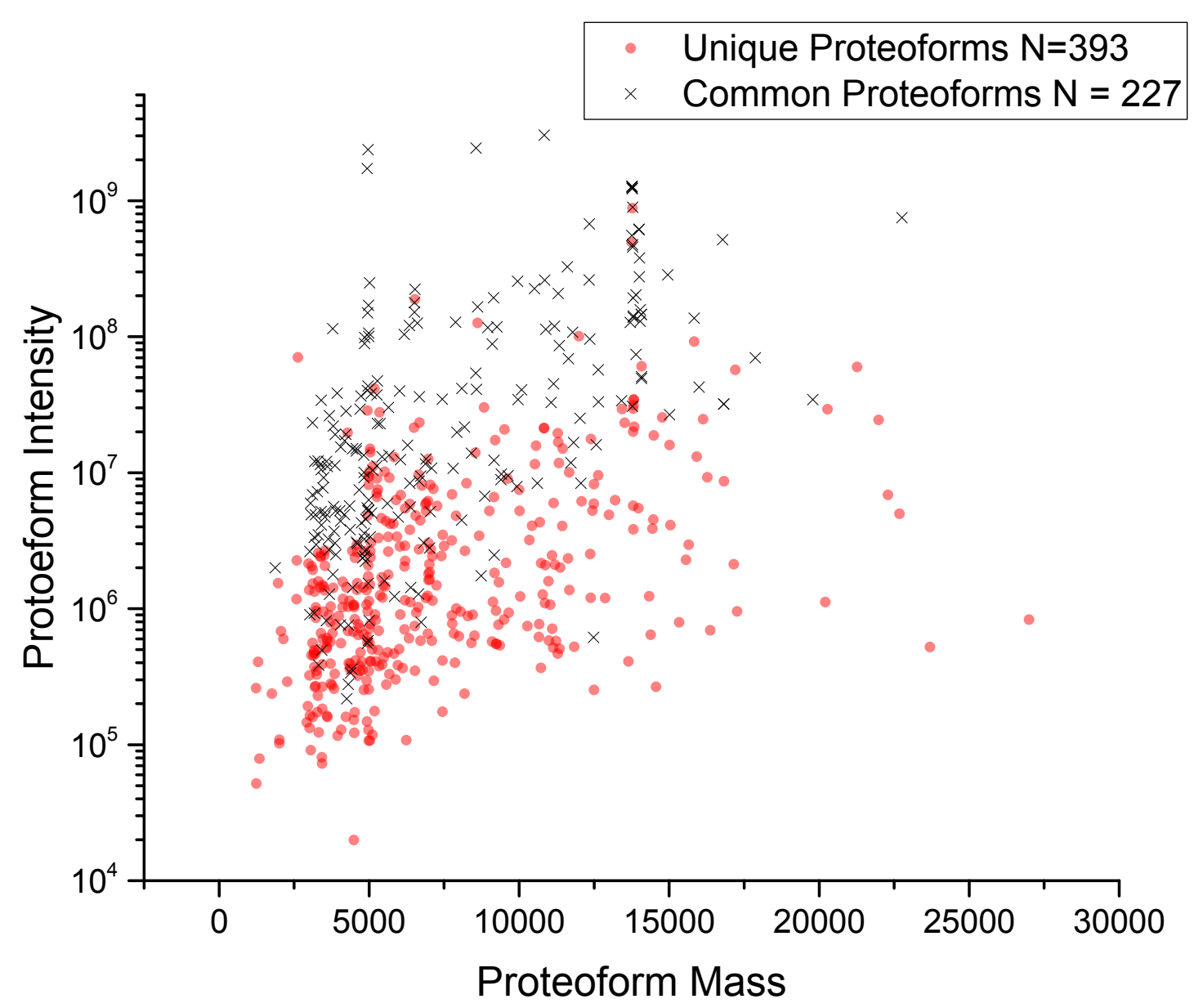

Figure S4. Mass and intensity distribution for proteoforms identified by triplicate nanoPOTS top-down analysis of $769 \pm 22$ cells. The black crosses indicate 227 common proteoforms identified for all cell loadings tested (i.e. shared with those detected from $74 \pm 7$ cells and $217 \pm 79$ cells) while the red dots represent 393 proteoforms uniquely identified starting with largest cell loading (i.e. $769 \pm 22$ cells). The proteoform intensities were corresponded to the "feature intensity" values in TopPIC results using the median of triplicate analysis of $769 \pm 22$ cells. The distribution of the unique proteoforms was biased towards lower intensities. 


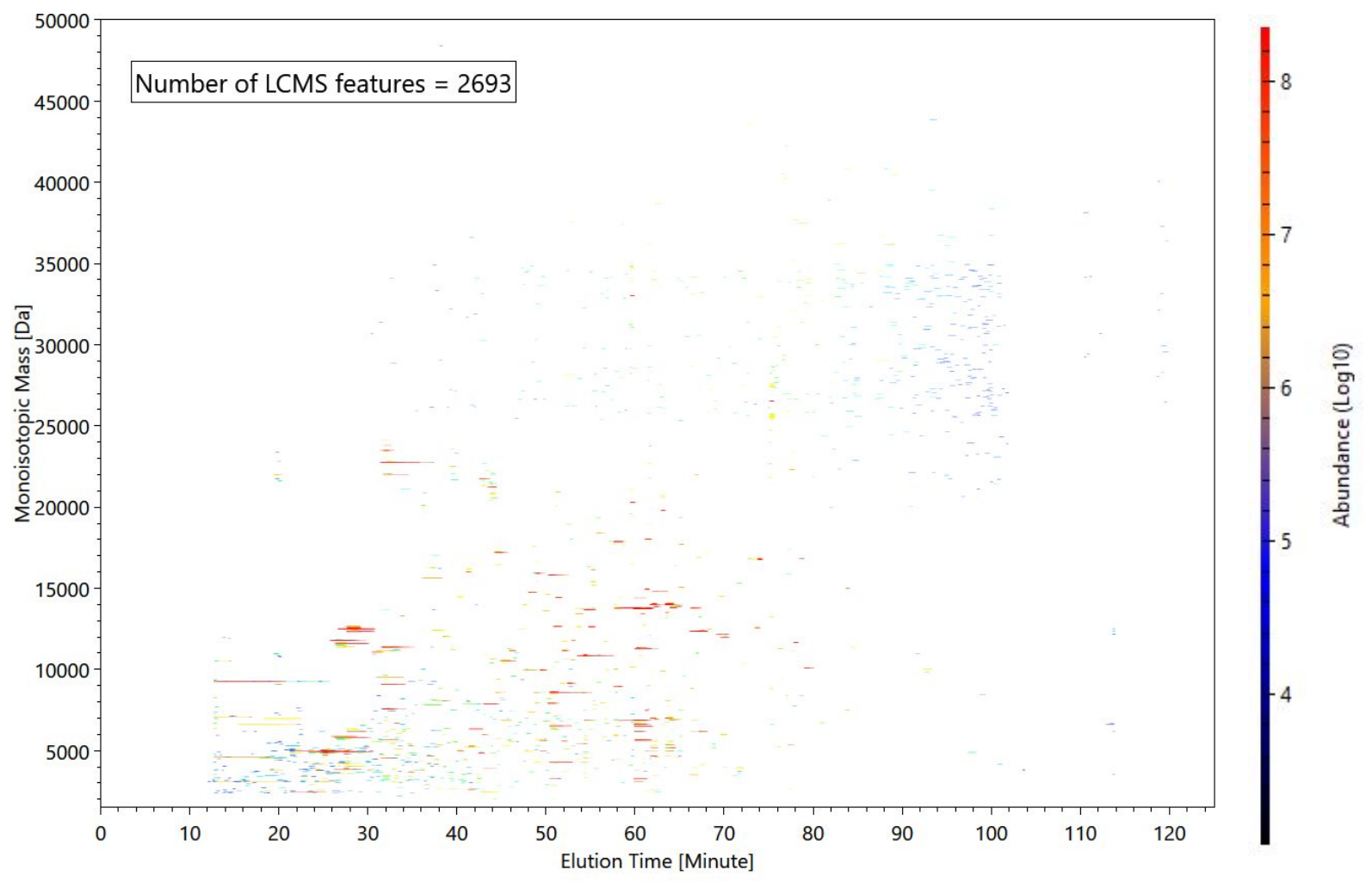

Figure S5. Representative feature map of nanoPOTS top-down analysis of $777 \mathrm{HeLa}$ cells produced by ProMex. The $\mathrm{x}$ axis corresponds to the LC elution time and the y corresponds to is the deconvoluted mass. The intensity is color coded as indicated by the scale on the right. Most of the isotopically resolved proteoforms are below $20 \mathrm{kDa}$ using the method and instrument described here. The features above $20 \mathrm{kDa}$ at $80-110 \mathrm{~min}$ are mostly deconvolution artifacts likely from the unresolved high mass proteins. 

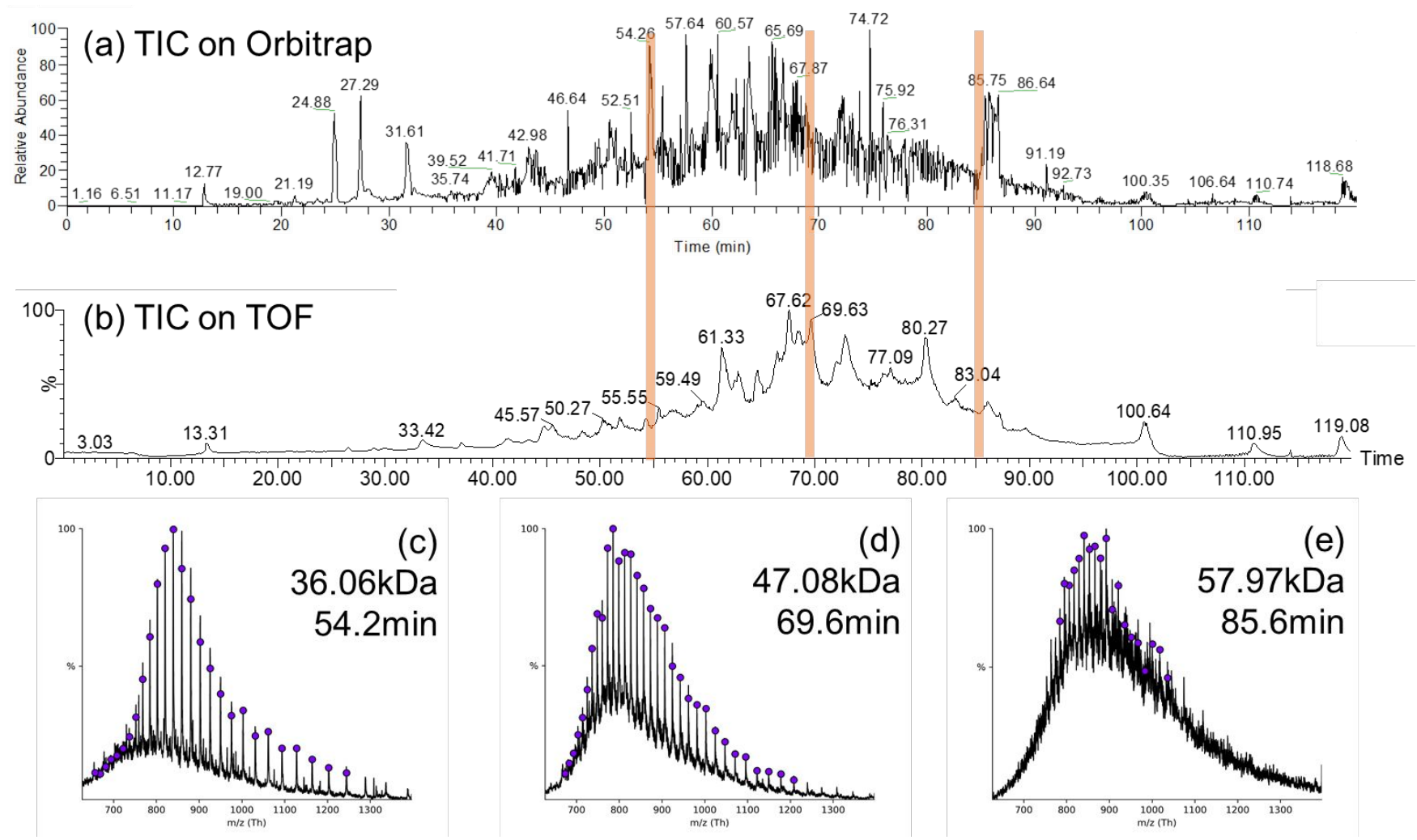

Figure S6. Total ion chromatogram of nanoPOTS top-down analysis using $\sim 760$ cells, and $0.1 \%$ $\mathrm{DDM}+8 \mathrm{M}$ urea in the extraction buffer. The data was acquired on Thermo Orbitrap Lumos (a) as described in the Experimental section. (b) Total ion chromatogram of a technical replicate using the same chromatography system on a Waters Synapt G2s-i TOF mass spectrometer. The inserts depict a few examples of larger proteins detected from the TOF MS. The TOF data was acquired using resolution mode and a $\mathrm{m} / \mathrm{z}$ range of 400-5000. Spectra were accumulated and saved every $5 \mathrm{~s}$. UniDec (https://github.com/michaelmarty/UniDec) was used to deconvolute non-isotopically resolved data. 


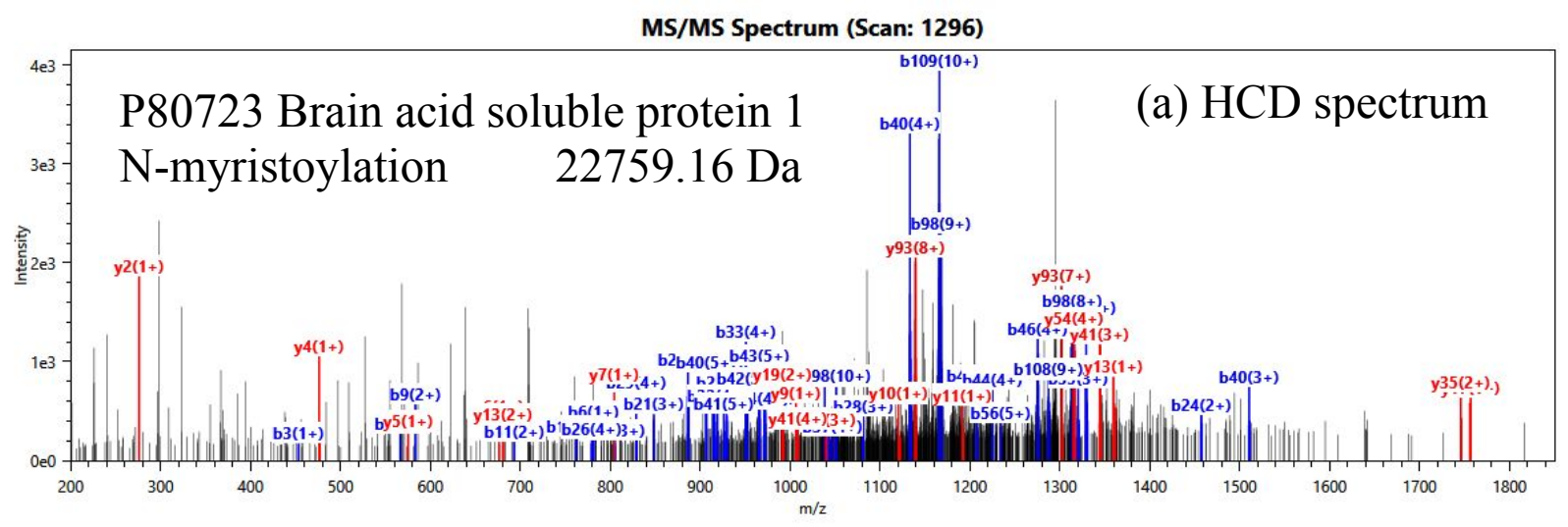

(b) Mass error map

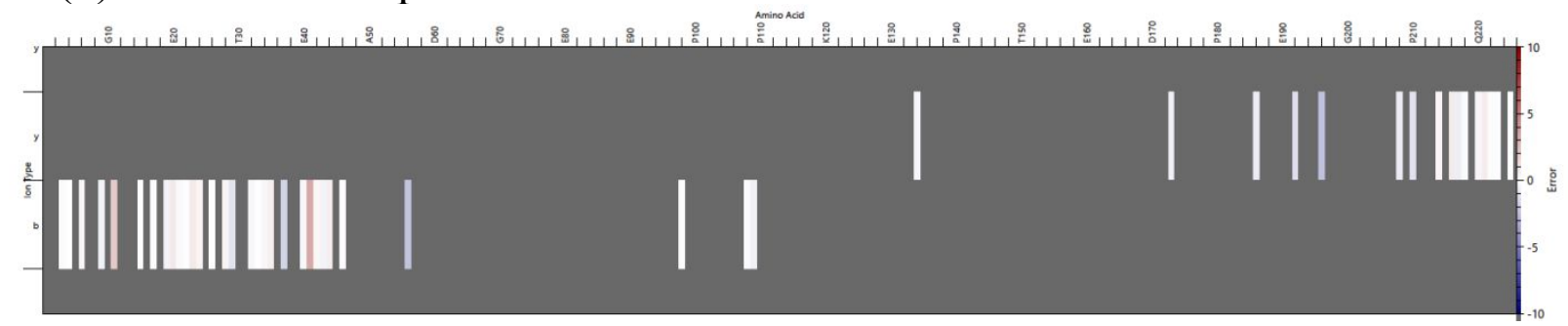

(c) Previous MS spectrum

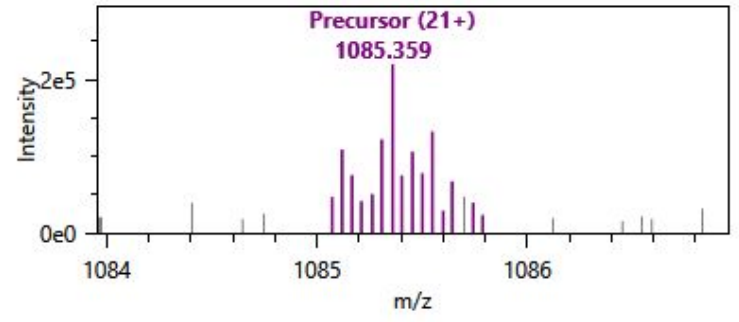

(d) Next MS spectrum

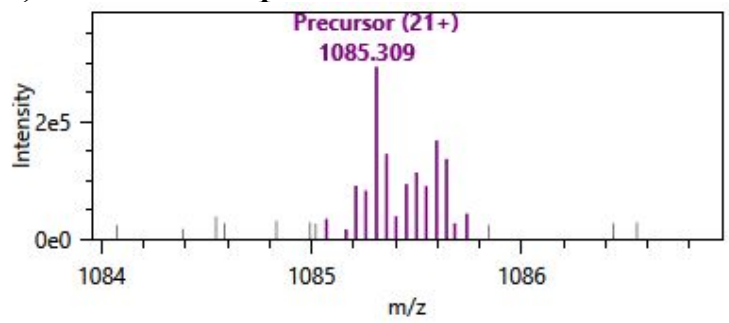

Figure S7. (a) HCD spectrum for the proteoform identified as N-myristoylated brain acid soluble protein (UniProt ID: P80723). An N-terminal mass addition of 210.198Da was detected. Nmyristoylation was assigned based on the knowledge about this modification in UniProt. (b) Mass error map for the matched fragments along the protein sequence in the horizonal axis. Top row denotes $\mathrm{y}$ and bottom row $\mathrm{b}$ ions. The color indicates mass error in ppm, with scale shown on the right edge of the map. While color represents mass errors near 0 ppm. (c-d) Spectra adjacent to the HCD spectrum in (a), show matched isotope peaks of the precursor. 


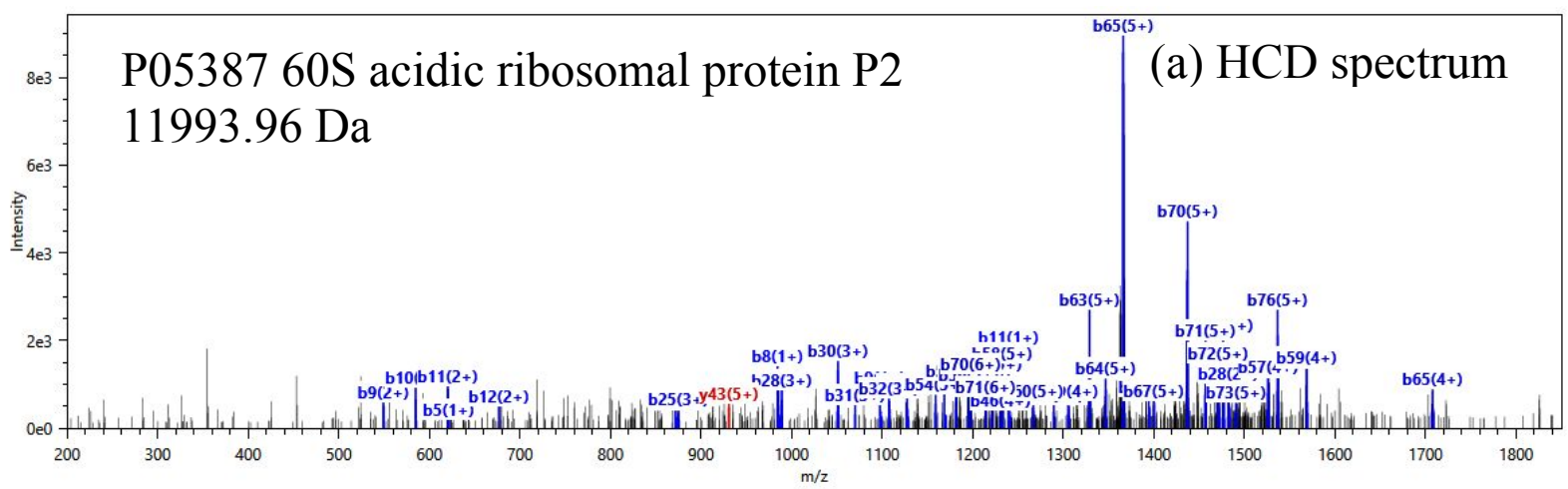

(b) Putative assignments of modifications

MRYVASYLLAALGGNSSPSAKDIKKIL DSVGIEADDDRLNKVISELNGKNIEDVI AQGIGKLASVPAGGAVAVSAAPGS[Ph ospho]AAPAAGS[Phospho]APAAAEEKK DEKKEES[Phospho]EES[Phospho]DDDM [Oxidation]GFGLFD

(d) Previous MS spectrum

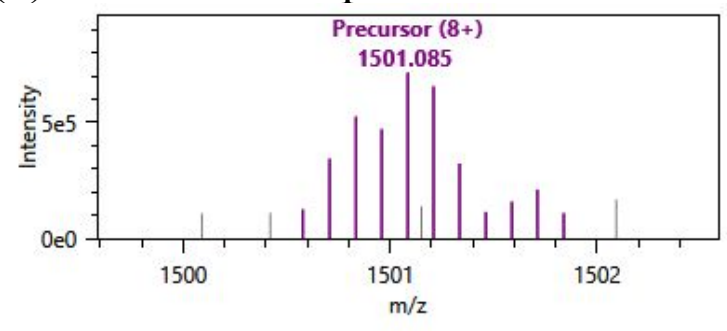

(c) Mass error map

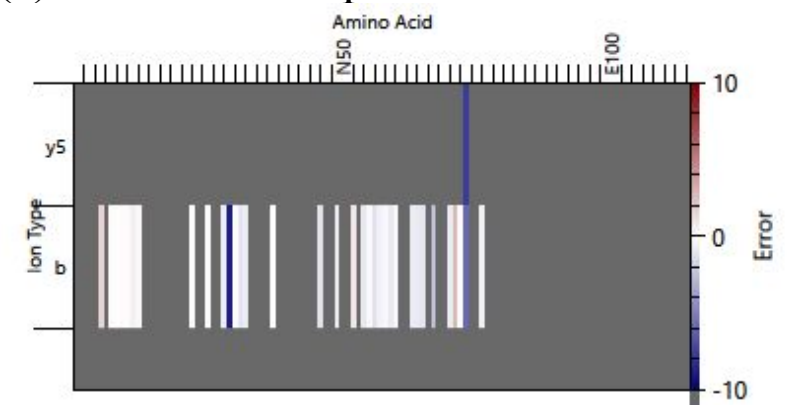

(e) Next MS spectrum

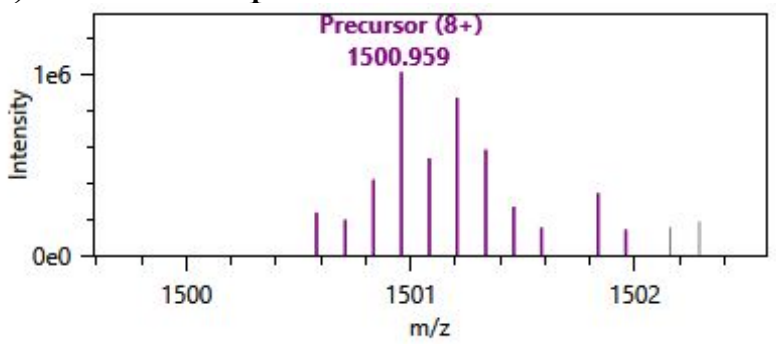

Figure S8. (a) HCD spectrum for the proteoform identified as $60 \mathrm{~S}$ acidic ribosomal protein $\mathrm{P} 2$ (UniProt ID: P05387). (b) Putatively assigned modifications (4 phosphorylation and 1 oxidation) based on the unknown mass shift of $335.8 \mathrm{Da}$ detected near the C-terminus. The protein has several known phosphorylation sites as noted in UniProt. (c) Mass error map for the matched fragments. The precursor peaks in the MS spectra before and after the HCD spectrum are shown in (d) and (e). 


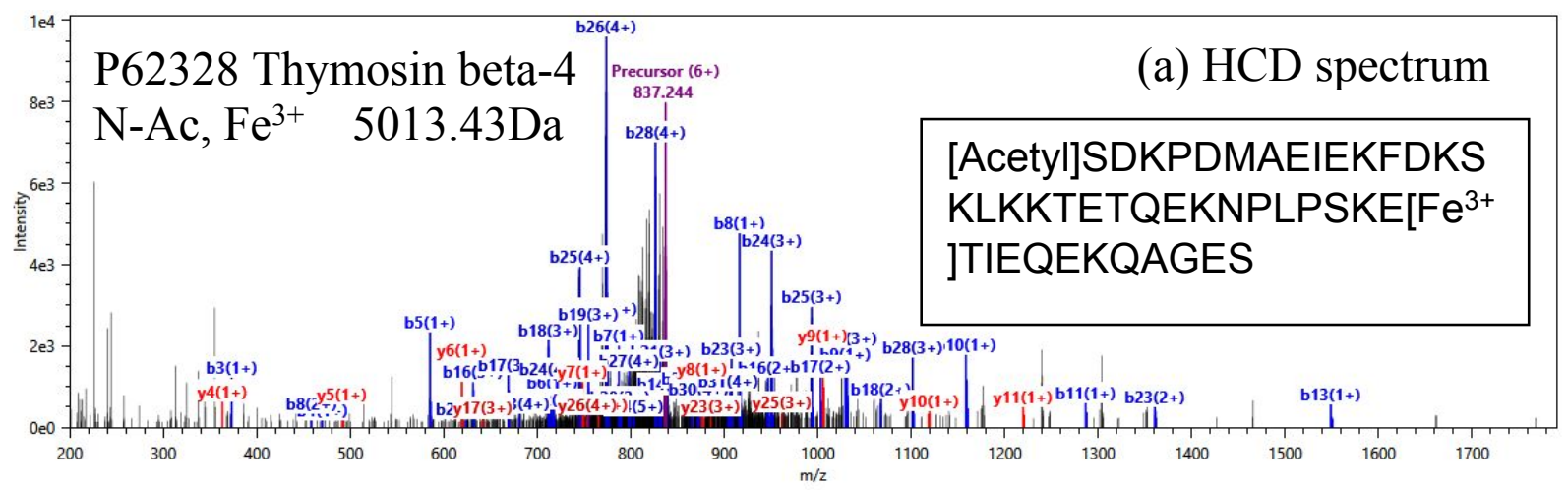

(b) Mass error map
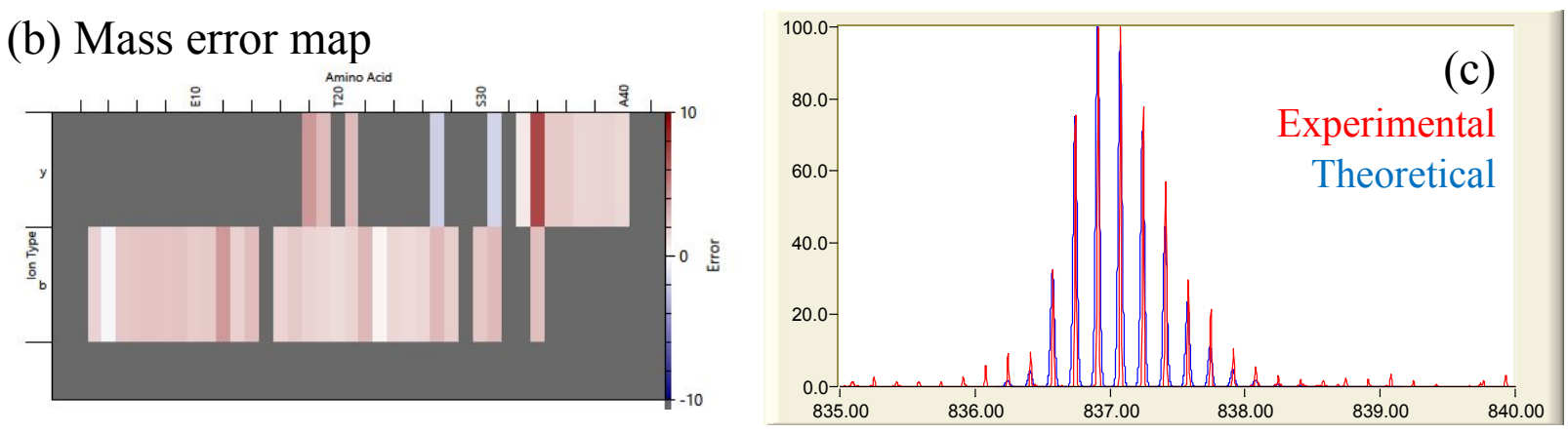

Figure S9. (a) HCD spectrum for the proteoform identified as N-acetylated and iron-bound thymosin beta-4 (UniProt ID: P62328). This assignment was based on detected mass shift of $52.92 \mathrm{Da}$ (mass of $\mathrm{Fe}^{3+}$ and substitution of 3 protons). (b) Mass error map for the matched fragments along the protein sequence on the horizonal axis. Top row corresponds to $y$, and bottom row to $\mathrm{b}$ ions. The color indicates mass error in ppm, with scale shown on the right edge of the map. (c) The experimental precursor spectrum (red) overlaid on the theoretical isotope distribution for $\mathrm{C}_{212} \mathrm{H}_{347} \mathrm{~N}_{56} \mathrm{O}_{78} \mathrm{~S}_{1} \mathrm{Fe}^{6+}$ (blue). 